\title{
As práticas da psicologia no contexto interdisciplinar no NASF: uma revisão sistemática
}

\section{The practices of psychology in the interdisciplinary context of NASF: systematic review}

\author{
Guilherme Severo Ferreira (orcid.org/0000-0003-3445-429X) ${ }^{1}$ \\ Gabriela Lemos de Pinho Zanardo (orcid.org/0000-0002-5705-7652)2 \\ Larissa Moraes Moro (orcid.org/0000-0001-7962-4577)33 \\ Marina Damion (orcid.org/0000-0002-8457-7979) ${ }^{4}$ \\ Kátia Bones Rocha (orcid.org/0000-000 1-7603-1709)5
}

\begin{abstract}
Resumo
Este estudo é uma revisão sistemática sobre práticas desenvolvidas pelos psicólogos no contexto interdisciplinar do Núcleo de Apoio à Saúde da Família (NASF). Efetivou-se a busca nas bases de dados PsycInfo, Scielo, Pubmed e BVS, utilizando o termo "NASF". Foram encontrados 551 artigos e, após refinada busca com os critérios de inclusão e exclusão, foram analisados 21 estudos. Os resultados foram organizados de acordo com as ações que envolviam os psicólogos, sendo o atendimento individual a principal atividade atribuída a eles. Dentre as ações desses profissionais que possuíam um caráter interdisciplinar, destaca-se: sensibilização da equipe para o apoio matricial e para acolhimento e humanização no cuidado. Apesar dos avanços da política, constatou-se dificuldade dos profissionais da atenção básica em aderir ao apoio matricial e realizar trabalho conjunto, seja pela resistência em superar o modelo biomédico ou pelo volume de trabalho e falta de tempo para cumprir diferentes tarefas.
\end{abstract}

\footnotetext{
${ }^{1}$ Pontifícia Universidade Católica do Rio Grande do Sul, Porto Alegre, Brasil. E-mail: guilherme.ferreiraa@gmail.com

2 Pontifícia Universidade Católica do Rio Grande do Sul, Porto Alegrei, Brasil. E-mail: gabrielazanardo@hotmail.com

3 Pontifícia Universidade Católica do Rio Grande do Sul, Porto Alegre, Brasil. E-mail: larissamoraesmoro@gmail.com

4 Pontifícia Universidade Católica do Rio Grande do Sul, Porto Alegre, Brasil. E-mail: ninah_d@hotmail.com

5 Pontifícia Universidade Católica do Rio Grande do Sul, Porto Alegre, Brasil. E-mail: katiabonesrocha@gmail.com
} 
Palavras-chave: Psicologia. Atenção primária à saúde. Apoio matricial. Revisão sistemática. NASF.

\begin{abstract}
This study is a systematic review about practices of psychologists of the Family Health Support Teams (NASF) in the interdisciplinary context. The search of the databases was performed in PsycINFO, SciELO, PubMed and BVS, using the term "NASF". We found 551 articles and after research refinement of inclusion and exclusion criteria, we analyzed 21 articles. Results were organized according to actions that evolved psychologists, which individual care was the main activity attributed to them. Among the actions of these professionals that had an interdisciplinary character, we emphasize the sensitization of the team for the support matrix and for reception and humanizing care. Despite the advances in the policy, primary care professionals showed difficulty in adhering to the matrix support and in conducting joint work, either due to the resistance in overcoming the biomedical model, or due to the workload and time constraints.
\end{abstract}

Keywords: Psychology. Primary care. Matrix support. Systematic review. NASF.

A atenção básica constitui-se como porta de entrada do Sistema Único de Saúde (SUS) e caracteriza-se como um conjunto de ações de saúde, desde a promoção em saúde até o diagnóstico e tratamento de doenças. Seus objetivos consistem em desenvolver atenção integral e continuada, realizar cuidado territorializado, estabelecer vínculo com a população atendida, bem como planejar e desenvolver ações baseadas nas demandas da comunidade (Brasil, 2011).

Com o objetivo de ampliar a abrangência e escopo das ações da atenção básica, foi criado, em 2008, o Núcleo de Apoio à Saúde da Família (NASF). Ele é composto por equipe multiprofissional que trabalha em conjunto com a Estratégia Saúde da Família (ESF) (Brasil, 2008). Como forma de expansão e consolidação da atenção, foi criada a Política Nacional de Atenção Básica (PNAB), que tem como estratégia prioritária a Saúde da Família (Brasil, 2011). Em 2017, foram revisadas as diretrizes do documento e, a partir disso, alterado o termo NASF para Núcleo Ampliado de Saúde da Família e AB (NASF-AB). A publicação atual apresenta como mudança mais expressiva a inclusão de prestação de serviços diretos à população, assim como ações de suporte clínico, pedagógico e sanitário (Brasil, 2017).

O NASF não se constitui como porta de entrada do sistema, à medida que atua de forma itinerante e integrada à ESF, equipes para populações específicas e academias da 
saúde. Além das novas atribuições, algumas das principais ações previstas são: discussão de casos; atendimento conjunto; construção de projetos terapêuticos; educação permanente; intervenções no território e na saúde de grupos populacionais e da coletividade; ações intersetoriais; ações de prevenção e promoção da saúde; discussão do processo de trabalho, entre outras (Brasil, 2011).

Assim como as atividades que serão desenvolvidas pelo NASF, a escolha dos profissionais que compõem cada equipe deve ser fundamentada nas necessidades dos territórios e populações atendidas. Dessa forma, devido à grande demanda da atenção à saúde mental, preconiza-se a inserção de ao menos um profissional da área no NASF, podendo ser psicólogo, psiquiatra ou terapeuta ocupacional (Brasil, 2008).

O NASF e a ESF possuem como atribuição proporcionar cuidado integral, articulando ações de prevenção e promoção à saúde com enfoque no trabalho interdisciplinar. Nesse sentido, a principal estratégia utilizada é o apoio matricial, que auxilia na organização e desenvolvimento do trabalho e tem como objetivo proporcionar uma retaguarda especializada à ESF (Brasil, 2008). Além disso, o apoio matricial se configura como método de trabalho que disponibiliza suporte técnico-pedagógico às equipes de referência (Campos \& Domitti, 2007; Campos, Figueiredo, Pereira, \& Castro, 2014). Assim, com o intuito de superar a prática de encaminhamento, com base em mecanismos de referência e contrarreferência, esse arranjo organizacional visa facilitar o direcionamento dos fluxos da rede. Por meio do compartilhamento de casos e discussões conjuntas, fomenta-se a produção de corresponsabilização entre as equipes (Campos et al., 2014; Dimenstein et al., 2009).

No contexto do NASF, não são encontradas funções determinadas para cada profissão nos documentos ministeriais (Brasil, 2008, 2011). Na literatura científica, foram encontradas duas revisões de literatura que analisam as ações de apoio matricial desenvolvidas na atenção básica (Bonfim, Bastos, Góis, \& Tófoli, 2013; Castro \& Campos, 2014).

Destaca-se a falta de definição sobre o papel da Psicologia no NASF, assim como pesquisas que enfatizem o trabalho que vem sendo realizado por esses profissionais. Vale 
ressaltar que muitos artigos sobre a Psicologia no NASF são estudos de caso que analisam apenas uma de suas atribuições cotidianas, faltando, assim, uma revisão de diferentes pesquisas para explorar quais são essas práticas e como vêm sendo executadas.

Considerando a recente implantação do NASF e a possibilidade de inclusão dos profissionais da psicologia no cenário da atenção básica, evidencia-se a importância de analisar quais ações vêm sendo realizadas no NASF. Sendo assim, foi realizada uma revisão sistemática sobre as práticas desenvolvidas por profissionais da Psicologia no contexto do NASF, com objetivo de analisar tanto as ações interdisciplinares, quanto as específicas dos psicólogos.

\section{Método}

O levantamento bibliográfico foi realizado nas bases de dados eletrônicas PsycINFo, SciELO, Pubmed e BVS. Nos campos de busca, foram inseridos os termos "NASF" e "NASF-AB" com o operador booleano "OR", e escolhida a opção "contém" a palavra (nos títulos, resumos ou texto), no intuito de integrar todas as pesquisas que realizaram investigações sobre esse dispositivo. Após algumas simulações com o acréscimo de outros descritores, como "psicologia" ou "interdisciplinar", optou-se por utilizar apenas os termos descritos porque os resultados das buscas mostraram-se muito restritos. Visto que a Portaria que instituiu o NASF foi publicada em 2008, a busca foi realizada com o recorte temporal, considerando publicações a partir de janeiro de 2008.

Dois juízes independentes realizaram as buscas, em agosto de 2019, e foram encontrados 551 artigos. Para refinar a busca, foram utilizados os seguintes critérios de inclusão: 1) tratar dos Núcleos de Apoio à Saúde da Família; 2) possuir psicólogos na equipe do NASF estudada. Justifica-se a escolha do segundo critério para analisar de forma ampla os artigos que apenas citam a participação desses profissionais, discutindo as ações da equipe de forma interdisciplinar, mas também os estudos que apresentam as práticas dos psicólogos de forma específica. Após a exclusão dos estudos repetidos, foi realizada a 
leitura integral dos 32 artigos incluídos e aplicados os critérios de exclusão: 1) artigos teóricos; 2) estudos não disponíveis em formato de artigo.

Em caso de discordância entre os juízes sobre a inclusão dos estudos, foi realizada a revisão e discussão do artigo, até ser alcançado um consenso. O banco de dados final contou com 21 estudos e o fluxograma sobre essa seleção encontra-se na Figura 1.

\section{Figura 1}

\section{Fluxograma}
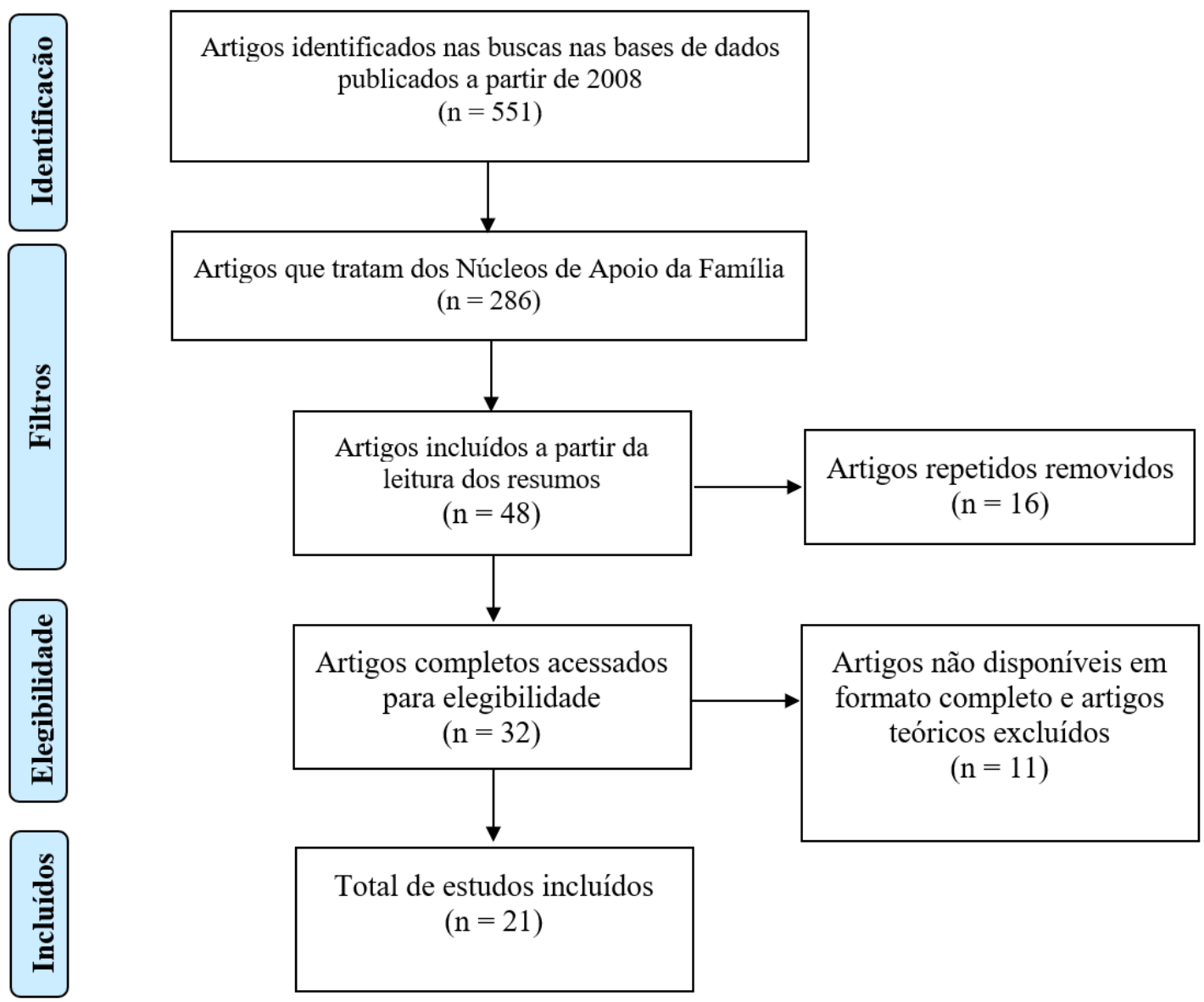

\section{Resultados}

A partir da leitura dos artigos na íntegra, foram extraídas as seguintes informações: autores, ano, local e participantes, que se encontram na Tabela 1. Os resultados foram organizados de acordo com as práticas que envolviam a participação de psicólogas, sendo 
elas: discussão de caso; atendimento conjunto; elaboração de Projeto Terapêutico Singular (PTS); visitas domiciliares; intervenções de promoção e prevenção de saúde e ações intersetoriais; atendimentos individuais; capacitação; registro de atividades; planejamento de ações; sensibilização da equipe e grupos e palestras psicoeducativas.

\section{Tabela 1}

Quadro resumo dos estudos

\begin{tabular}{|c|c|c|c|c|}
\hline & Autores & Ano & Estado & Participantes \\
\hline 1 & Sundfeld & 2010 & RJ & uma psicóloga do NASF \\
\hline 2 & Santeiro & 2012 & GO & $\begin{array}{l}\text { estagiários de psicologia de } \\
\text { NASF }\end{array}$ \\
\hline 3 & Azevedo \& Kind & 2013 & MG & sete psicólogas do NASF \\
\hline$\overline{4}$ & Lancman et al. & 2013 & SP & $\begin{array}{l}\text { dois NASF (contavam com } 15 \\
\text { profissionais) }\end{array}$ \\
\hline 5 & Leite et al. & 2013 & RJ & seis psicólogas de NASF \\
\hline 6 & Ferro et al. & 2014 & PR & $\begin{array}{l}\text { oito profissionais da ESF e } \\
\text { quatro profissionais do NASF }\end{array}$ \\
\hline 7 & Hori \& Nascimento & 2014 & SP & $\begin{array}{l}\text { treze profissionais do NASF e } \\
\text { NAAB }\end{array}$ \\
\hline 8 & Moura \& Luzio & 2014 & PR & $\begin{array}{l}\text { sete profissionais de uma } \\
\text { equipe NASF }\end{array}$ \\
\hline 9 & Ribeiro et al. & 2014 & $\mathrm{PI}$ & $\begin{array}{l}\text { setenta e seis profissionais da } \\
\text { ESF }\end{array}$ \\
\hline 10 & Barros et al. & 2015 & SP & $\begin{array}{l}\text { vinte e dois profissionais de } \\
\text { duas equipes NASF }\end{array}$ \\
\hline 11 & Cela \& Oliveira & 2015 & $\mathrm{RN}$ & sete psicólogos do NASF \\
\hline 12 & Gonçalves et al. & 2015 & SP & $\begin{array}{l}\text { coordenação, equipe técnica e } \\
\text { trabalhadores do NASF }\end{array}$ \\
\hline 13 & Santana et al. & 2015 & PB & $\begin{array}{l}\text { dezenove enfermeiros que } \\
\text { atuam na ESF }\end{array}$ \\
\hline 14 & Sousa et al. & 2015 & RN & $\begin{array}{l}\text { três psicólogos e dois } \\
\text { coordenadores de dois NASF }\end{array}$ \\
\hline 15 & Soares \& Oliveira & 2016 & SC & $\begin{array}{l}\text { doze profissionais da equipe } \\
\text { NASF, um gerente distrital da }\end{array}$ \\
\hline
\end{tabular}




\begin{tabular}{lllll}
\hline & & & \multicolumn{1}{c}{$\begin{array}{l}\text { atenção primária e um gerente } \\
\text { distrital de saúde mental }\end{array}$} \\
\hline 16 & Macedo et al. & 2016 & CE & $\begin{array}{l}\text { onze profissionais de três } \\
\text { equipes NASF }\end{array}$ \\
\hline 17 & Santos et al. & 2017 & SP & $\begin{array}{l}\text { trinta e quatro profissionais de } \\
\text { cinco NASF e quarenta e quatro } \\
\text { profissionais de quarenta ESF }\end{array}$ \\
\hline 18 & Arce \& Teixeira & 2017 & BA & $\begin{array}{l}\text { doze profissionais de nove } \\
\text { equipes NASF }\end{array}$ \\
\hline 19 & Klein \& d'Oliveira & 2017 & SP & $\begin{array}{l}\text { oito psicólogos de equipes } \\
\text { NASF }\end{array}$ \\
\hline 20 & Oliveira et al. & 2017 & RN & $\begin{array}{l}\text { vinte e quatro psicólogos de } \\
\text { equipes NASF }\end{array}$ \\
\hline 21 & Vasconcelos \& & 2019 & PE & 15 psicólogos de equipes NASF \\
& Aléssio & & & \\
\hline
\end{tabular}

Em relação às discussões de caso realizadas entre NASF e ESF, sete artigos apresentam a ocorrência de discussões aprofundadas entre as equipes (Arce \& Teixeira, 2017; Barros, Gonçalves, Kaltner, \& Lancman, 2015; Gonçalves et al., 2015; Klein \& d'Oliveira, 2017; Lancman, Gonçalves, Cordone, \& Barros, 2013; Soares \& Oliveira, 2016; Sundfeld, 2010). Essas são descritas como um espaço de troca de experiências e partilha de conhecimento, no qual realiza-se avaliação de casos para encaminhamento e identificação de necessidades prioritárias.

No entanto, oito estudos apontam barreiras para a realização de discussão de caso (Barros et al., 2015; Cela \& Oliveira, 2015; Ferro, Silva, Zimmermann, Castanharo, \& Oliveira, 2014; Gonçalves et al., 2015; Hori \& Nascimento, 2014; Lancman et al., 2013; Santana, Azevedo, Reichert, Medeiros, \& Soares, 2015; Vasconcelos \& Aléssio, 2019). Um artigo identificou que o tempo de trabalho de ambas as equipes, ESF e NASF, era insuficiente para discussões aprofundadas, enquanto dois estudos apresentam que essa atividade ocupava a maior parte da jornada de trabalho dos profissionais, que indicavam falta de tempo para realizar outras tarefas previstas (Gonçalves et al., 2015; Lancman et al., 2013). 
Um estudo assinalou que a discussão de caso ocorria apenas quando a ESF necessitava de auxílio na condução das ações (Cela \& Oliveira, 2015). Outro aponta que a ausência de profissionais nas reuniões dificultava a realização de trocas e o vínculo entre as equipes (Hori \& Nascimento, 2014). Ainda, Ferro, Silva, Zimmermann, Castanharo e Oliveira (2014) observavam que os profissionais da ESF não reconheciam as reuniões com o NASF como um espaço para discussões.

Em relação ao atendimento conjunto, compreende-se como uma atividade realizada com usuário ou grupo por ao menos um profissional de cada equipe - ESF e NASF (Brasil, 2010). Em um estudo, o compartilhamento acontecia apenas entre trabalhadores do NASF (Cela \& Oliveira, 2015). Destaca-se que em apenas cinco artigos são apresentados atendimentos compartilhados entre as equipes NASF e ESF (Arce \& Teixeira, 2017; Barros et al., 2015; Gonçalves et al., 2015; Klein \& d'Oliveira, 2017; Oliveira et al., 2017), sendo que um deles aponta uma articulação ainda frágil, já que na maioria das vezes os encontros eram realizados por somente um profissional, abordando tema de sua área. Em duas pesquisas, é relatado que os profissionais da ESF mencionam que apesar de existir um trabalho conjunto, eles preferem o modelo de atendimento de caráter individual (Soares \& Oliveira, 2016; Vasconcelos \& Aléssio, 2019).

Outra atividade realizada pelos profissionais do NASF é a formulação do PTS, elaborado na perspectiva interdisciplinar, a partir de uma avaliação compartilhada entre profissionais da equipe e usuários/famílias. É construído levando em consideração os múltiplos saberes e a valorização do conhecimento do usuário na formulação da proposta terapêutica. Desse modo, estimula-se o vínculo entre usuário e equipe, por meio de um processo de corresponsabilização do cuidado (Pinto et al., 2011).

Em cinco estudos, os autores indicaram que não ocorria a construção de PTS (Ferro et al., 2014; Gonçalves et al., 2015; Hori \& Nascimento, 2014; Leite, Andrade, \& Bosi, 2013; Sundfeld, 2010). Uma pesquisa constatou que o termo não era utilizado pelos profissionais (Ferro et al., 2014), enquanto outra avaliou que o conceito de PTS era pouco conhecido pela equipe ESF e também por alguns profissionais do NASF (Gonçalves et al., 2015). Ainda, 
Sundfeld (2010) refere que apenas o NASF se envolvia na construção do PTS, não havendo participação conjunta da ESF e dos usuários. Na mesma direção, Leite, Andrade e Bosi (2013) indicam que a dificuldade de integração entre ESF e NASF impedia o planejamento terapêutico dos usuários. Apenas quatro pesquisas de fato mencionaram o desenvolvimento de PTS realizado por toda equipe (Azevedo \& Kind, 2013; Barros et al., 2015; Klein \& d'Oliveira, 2017; Moura \& Luzio, 2014).

Doze estudos citam como práticas do NASF a visita domiciliar (VD) (Arce \& Teixeira, 2017; Azevedo \& Kind, 2013; Barros et al., 2015; Gonçalves et al., 2015; Leite et al., 2013; Macedo, Guimarães, Sampaio, Morais, \& Carneiro, 2016; Oliveira et al., 2017; Ribeiro et al., 2014; Santana et al., 2015; Santos, Figueiredo, \& Lima, 2017; Sousa, Oliveira, \& Costa, 2015; Sundfeld, 2010). Segundo Azevedo e Kind (2013), as VD eram planejadas e conduzidas coletivamente, com a presença de um Agente Comunitário de Saúde (ACS) e dois profissionais de áreas distintas. As visitas eram desenvolvidas frequentemente e estavam voltadas para o atendimento daqueles que se encontram impossibilitados de irem ao serviço.

Conforme Gonçalves et al. (2015), as equipes dos NASF realizavam VD conforme as necessidades das ESF e da população, possuindo frequência flutuante. A partir da reunião e dos acordos entre NASF e ESF, compartilhavam-se as responsabilidades sobre as atividades, dentre elas as VD. Os profissionais acreditam que essa configuração fortalecia planejamentos e ações mais organizadas, priorizando o trabalho em equipe.

Os estudos de Leite et al. (2013) e Sundfeld (2010) abordam a importância da presença dos ACS na visita, pois auxiliava no estabelecimento do vínculo e na receptividade das famílias visitadas, já que fazem parte da comunidade. Oliveira et al. (2017) referem a realização de VD de profissionais do NASF em conjunto com ACS, sendo as visitas voltadas às especificidades do profissional, no caso da psicologia, questões de saúde mental, atendendo demandas específicas e emergenciais dos usuários ao invés de possuírem um caráter preventivo, de busca ativa e de acompanhamento. Em contrapartida, os profissionais entrevistados por Leite et al. (2013) indicaram dificuldade em criar laços com a área atendida, já que a equipe do NASF passava apenas um turno semanal em cada ESF apoiada. 
As atividades coletivas apareceram comumente associadas às ações de promoção e prevenção de saúde. Um exemplo são as intervenções no território e na saúde de grupos específicos da população (Arce \& Teixeira, 2017; Azevedo \& Kind, 2013; Barros et al., 2015; Gonçalves et al., 2015; Klein \& d'Oliveira, 2017; Moura \& Luzio, 2014; Oliveira et al., 2017; Ribeiro et al., 2014; Santana et al., 2015, Santos et al., 2017), nas quais destacam-se: grupo de qualidade de vida, nutrição, dança, postura (Azevedo \& Kind, 2013); grupo de hipertensos, diabéticos, gestantes, adolescentes, idosos, cuidadores, pessoas com sofrimento psíquico e uso de psicotrópicos, obesidade, grupo equilíbrio (realizado com os próprios profissionais de saúde) (Macedo et al., 2016; Moura \& Luzio, 2014; Oliveira et al., 2017); grupos terapêuticos e educativos, oficinas temáticas (Barros et al., 2015; Gonçalves et al., 2015, Oliveira et al, 2017); grupo de saúde mental (Gonçalves et al., 2015).

Dentre as práticas de grupo descritas, Leite et al. (2013) referem que os profissionais da psicologia eram os responsáveis em relação ao grupo conduzido com adolescentes, que tratava de temáticas como sexualidade, gravidez na adolescência e uso de drogas, e também de grupo psicoeducativo com adultos que se automedicam e fazem uso inadequado de psicofármacos.

Três estudos indicaram que o NASF era responsável pela coordenação dos grupos e/ou palestras socioeducativas, de responsabilidade apenas dos psicólogos (Gonçalves et al., 2015; Lancman et al., 2013; Oliveira et al., 2017). Os motivos elencados foram a elevada demanda de atendimento para ESF (Gonçalves et al., 2015) e a resistência de alguns profissionais da Estratégia em pactuar ações com o NASF, somadas às variadas formas de organização do trabalho entre as equipes (Lancman et al., 2013).

As ações intersetoriais são apontadas em nove estudos, por meio da articulação com a rede de serviços de saúde, assistência, educação e cultura (Arce \& Teixeira, 2017; Azevedo \& Kind, 2013; Barros et al., 2015; Gonçalves et al., 2015; Moura \& Luzio, 2014; Oliveira et al., 2017; Ribeiro et al., 2014; Santana et al., 2015; Soares \& Oliveira, 2016). Alguns exemplos: discussões e acompanhamento conjunto de casos com serviços da Assistência Social e Conselho Tutelar (Cela \& Oliveira, 2015); articulação com Conselho Tutelar, Centro 
de Atenção Psicossocial (CAPS), ambulatório de especialidades, entre outros (Gonçalves et al., 2015; Oliveira et al., 2017); participação no Programa Saúde na Escola (Arce \& Teixeira, 2017; Oliveira et al., 2017; Santana et al., 2015) e exploração dos recursos da comunidade (Soares \& Oliveira, 2016).

Dentre os 21 estudos analisados, somente quatro não abordavam as práticas específicas de cada profissional no NASF (Arce \& Teixeira, 2017; Hori \& Nascimento, 2014; Moura \& Luzio, 2014; Soares \& Oliveira, 2016). Em relação ao trabalho desenvolvido por psicólogos, a principal atividade identificada foi o atendimento individual, relatado em 12 estudos (Azevedo \& Kind, 2013; Barros et al., 2015; Cela \& Oliveira, 2015; Ferro et al., 2014; Gonçalves et al., 2015; Klein \& d'Oliveira, 2017; Lancman et al., 2013; Leite et al., 2013; Oliveira et al., 2017; Santana et al., 2015; Santeiro, 2012; Sousa et al., 2015; Sundfeld, 2010).

No estudo de Sundfeld (2010), são apresentadas solicitações de atendimento pela escola, Conselho Tutelar, pais e equipe de saúde, por exemplo: queixas de agressividade na escola, problemas de relacionamento familiar e dificuldades de aprendizagem. No mesmo sentido, são mencionadas procuras feitas pelos usuários, que relatavam sintomas depressivos e crises nervosas.

Já Azevedo e Kind (2013) referem que os atendimentos individuais realizados pelas psicólogas não se configuravam como psicoterapia. Os atendimentos tinham entre seis a oito sessões e eram realizados com usuários atendidos por outros profissionais que identificaram questões de ordem psicológica, como crianças com dificuldades de aprendizagem, idosos com demência, entre outros. Em alguns casos, o atendimento acontecia em um encontro em que eram realizados apontamentos e orientações ao usuário (Azevedo \& Kind, 2013).

No estudo de Klein e d'Oliveira (2017), os psicólogos relataram que a possibilidade de intervenção específica de um técnico é vista por eles como descaracterização da proposta do apoio matricial e do NASF. No entanto, identificaram que esse atendimento é uma demanda constante da equipe ESF. 
Os outros onze artigos encontraram que os NASF acabam atuando como ambulatórios especializados (Barros et al., 2015; Cela \& Oliveira, 2015; Ferro et al., 2014; Gonçalves et al., 2015; Lancman et al., 2013; Leite et al., 2013; Oliveira et al., 2017; Santana et al., 2015; Santeiro, 2012; Sousa et al., 2015; Vasconcelos \& Aléssio, 2019). Os trabalhadores da ESF identificam e encaminham as demandas aos profissionais do NASF e os próprios usuários solicitam esse tipo de atendimento. Oliveira et al. (2017) especificam um tipo de atendimento individual caracterizado como acolhimento, em que os profissionais ofertavam escuta qualificada aos usuários que apresentassem ao serviço uma demanda de atendimento especializado, sendo feitas orientações e encaminhamentos. Porém, em alguns dos contextos estudados, os profissionais do NASF possuíam discernimento sobre a proposta de trabalho da equipe, apesar das solicitações da ESF e da população (Barros et al., 2015; Cela \& Oliveira, 2015; Klein \& d'Oliveira, 2017; Leite et al., 2013; Sousa et al., 2015). Dessa forma, eles conseguiam operacionalizar essas demandas a partir de outra configuração de cuidado (Barros et al., 2015; Leite et al., 2013).

Dois estudos apontam como trabalho do psicólogo a realização de capacitação em saúde mental para com os ACS, voltada para melhor identificação desses casos na comunidade e planejamento de formas atuação (Leite et al., 2013; Oliveira et al., 2017). Além disso, percebeu-se que o espaço teve efeito terapêutico, já que os agentes aproveitaram esse ambiente para falar sobre as angústias sentidas frente às questões psicossociais relacionadas à pobreza, violência e drogas.

Oliveira et al. (2017) pontuam o registro de atividades e planejamento de ações como atividades executadas por psicólogos. A primeira com caráter de prestação de contas, exigida pelas Secretarias de Saúde, por meio de relatórios de atividades e fichas de produtividade. Já a segunda diz respeito a conhecer o perfil epidemiológico e demanda das comunidades atendidas para aperfeiçoar as ações realizadas no território. Porém este trabalho acabava acontecendo de forma descontextualizada e isolada por alguns profissionais, sem espaço para discussão e construção coletiva. 
Três artigos abordam como contribuição da psicologia a sensibilização e conscientização das equipes da atenção básica para o modelo de trabalho baseado no apoio matricial (Cela \& Oliveira, 2015; Klein \& d'Oliveira, 2017), bem como questões como o acolhimento e humanização do cuidado (Leite et al., 2013). Os entrevistados entendem que essa mudança é necessária e que, assim, pode-se "potencializar uma transformação das interações entre profissionais, usuários e comunidade" (Leite et al., 2013, p. 1175). Em uma dessas pesquisas, os psicólogos relatam mudanças no olhar dos profissionais das equipes de saúde da família no reconhecimento da subjetividade e na desmitificação de problemas de saúde mental (Klein \& d'Oliveira, 2017).

\section{Discussão}

A proposta de trabalho do NASF é superar o paradigma convencional de prestação de cuidados em saúde, em que prevalece uma assistência curativista, especialista e fragmentada (Perrella, 2015). Assim, segue-se em direção à corresponsabilização e gestão integrada do cuidado, priorizando atendimentos compartilhados e projetos terapêuticos que incluam a participação do usuário, considerando sua singularidade (Nascimento \& Oliveira, 2010). Esses fundamentos balizadores devem perpassar todas as práticas realizadas pelo NASF, caso contrário, as ações baseadas nessas diretrizes de cuidado ficam comprometidas.

No entanto, observa-se que as orientações dos documentos ministeriais (Brasil, 2008, 2011) sobre as práticas dos profissionais do NASF possuem um caráter amplo e, por vezes, superficial sobre o tema em questão, o que possibilita diferentes interpretações e margem para múltiplas condutas. Isso ressalta a importância de conhecer as práticas que vêm sendo desenvolvidas pela equipe do NASF, principalmente no que tange ao fazer do psicólogo, que pela primeira vez se insere na equipe básica nesse nível de atenção.

Nessa busca, foram encontradas práticas heterogêneas em demasia, o que dificulta uma análise comparativa do funcionamento dos serviços. Cabe destacar que as atividades podem ser distintas devido às necessidades específicas de cada território, porém, elas devem ser pautadas nos princípios do SUS e adequadas ao contexto da atenção básica. 
Esse achado está de acordo com o que apontam Correia, Goulart e Furtado (2017) sobre as discrepâncias entre a Portaria que dá origem ao NASF e os Cadernos de Atenção Básica (Brasil, 2008, 2010). A primeira possui um caráter genérico em relação às atribuições dos profissionais, enquanto que o segundo apresenta detalhadamente as diretrizes e ações a serem desenvolvidas. Como consequência, constituíram-se diferentes práticas nas equipes do NASF, gerando particularidades, inclusive, entre Núcleos de um mesmo município.

No tocante às práticas da Psicologia, a principal atividade exposta foi o atendimento individual, que na maioria dos casos era baseado na clínica tradicional, numa lógica ambulatorial especializada. A partir de uma atuação individualista e fragmentada, essa prática compromete a dinâmica interdisciplinar. De acordo com Sousa, Oliveira e Costa (2015), o atendimento clínico desvinculado de outras atividades da ESF se opõe ao caráter interdisciplinar da política. Cunha e Campos (2011) ressaltam que os profissionais podem fazer atendimentos individuais, todavia, essa atividade não deve ser prioritária.

Segundo os Cadernos da Atenção Básica (Brasil, 2014), o atendimento individual não é vetado pela lógica do apoio matricial. A frequência de atendimentos individuais específicos pode variar conforme a modalidade do NASF, o número de equipes apoiadas e a insuficiência de serviços assistenciais, já que o NASF deve contribuir para a resolutividade dos casos. Porém, não exclui a responsabilidade dos municípios em investir nos serviços de saúde necessários para atender essas demandas.

Em contrapartida, identificou-se que alguns psicólogos buscavam problematizar os pedidos trazidos pela comunidade e pela ESF por atendimento no modelo ambulatorial. Além disso, aponta-se que os usuários atendidos pelos psicólogos também eram acompanhados por outros profissionais, colocando em prática a corresponsabilização dos casos (Barros et al., 2015; Cela \& Oliveira, 2015; Klein \& d'Oliveira, 2017; Leite et al., 2013; Sousa et al., 2015, Sundfeld, 2010). Conforme Silva et al. (2012), durante a implementação do NASF, as equipes devem compartilhar conhecimentos e experiências, configurando uma relação multidisciplinar. Com a crescente apropriação do território e dos casos atendidos, assim 
como a maior integração entre as equipes, espera-se que novos saberes sejam construídos, percorrendo as diferentes especialidades de uma forma dinâmica.

Dessa forma, objetiva-se que as atividades e discussões não sejam de responsabilidade exclusiva de um profissional, por exemplo, deixar a cargo do psicólogo os casos de saúde mental, por ter como fundamento a escuta terapêutica (Perrella, 2015). Apesar de estar preconizada a presença de no mínimo um profissional de saúde mental no NASF (Brasil, 2008), é importante atentar para que essa designação não acarrete a fragmentação do cuidado (Ribeiro et al., 2014). Sendo assim, espera-se que as ações sejam desenvolvidas de forma interdisciplinar entre a equipe matriciadora e a equipe de referência.

Nesse sentido, de forma geral, percebeu-se que diferentes profissionais da ESF apresentavam dificuldade em aderir à proposta de apoio matricial do NASF. Os estudos apontam que muitas equipes não conseguem superar a lógica tradicional, sob viés biomédico (Barros et al., 2015; Cela \& Oliveira, 2015; Ferro et al., 2014; Gonçalves et al., 2015; Klein \& d'Oliveira, 2017; Lancman et al., 2013; Leite et al., 2013; Oliveira et al., 2017; Santana et al., 2015; Santeiro, 2012; Sousa et al., 2015; Vasconcelos \& Aléssio, 2019). Alguns dos motivos apresentados pelos profissionais da ESF foram: a resistência em desenvolver trabalho conjunto (compartilhamento de experiências e espaços de reuniões), o grande volume de trabalho e a falta de tempo para dar conta de diferentes tarefas. Essas razões, somadas à solicitação dos usuários por consultas individuais especializadas, resultavam na prática de atendimentos individuais pelos profissionais do NASF. Entende-se que essa forma de atendimento não se configura como conjunta por não ocorrer conexão entre equipe apoiadora e equipe de referência. Sendo assim, observa-se que ao invés de desenvolverem ações por meio da corresponsabilização dos casos, as ESF delegavam suas responsabilidades às equipes NASF.

Além disso, profissionais do NASF justificavam a falta de equipamentos e profissionais especializados para dar conta dessa demanda (Oliveira et al., 2017), o que está de acordo com outros autores que reforçam que limitações estruturais, como a falta de serviços especializados, induzem a ESF a solicitar indevidamente que os profissionais do NASF atendam de forma substitutiva, ao invés da ótica do apoio matricial (Cunha \& Campos, 
2011 ; Nascimento \& Oliveira, 2010; Silva et al., 2012). Isto posto, um dos desafios do NASF, segundo Silva et al. (2012), é articular as atividades com a agenda da ESF, considerando o cotidiano de trabalho e as metas estabelecidas, redimensionando o tempo utilizado. Para transformar ações é preciso que ocorram mudanças nos paradigmas instituídos nos serviços da atenção básica e nas relações interdisciplinares. É a partir do diálogo e do contato entre práticas e concepções de atenção que se promoverá uma nova forma de trabalho em saúde (Nascimento \& Oliveira, 2010).

Nesse ponto, destaca-se a relevância do profissional da psicologia nessas equipes, como ponte para auxiliar na mudança dessa perspectiva. Os psicólogos possuem conhecimentos que podem propiciar uma atuação com olhar atento para as inter-relações entre o contexto e as singularidades, além de compreender a dinâmica dos fenômenos envolvidos nesse cenário de práticas. Alguns exemplos seriam os desdobramentos a partir da discussão dos processos de trabalho, seja na possibilidade de sensibilizar a equipe para a atuação baseada no apoio matricial ou na abordagem de questões como acolhimento e humanização no cuidado (Cela \& Oliveira, 2015; Klein \& d'Oliveira, 2017; Leite et al., 2013). Essa prática seria uma alternativa potente para efetivar a mudança na concepção de saúde e de trabalho das equipes da atenção básica.

Discutir as relações e processos de trabalho auxilia na identificação de dificuldades, no estabelecimento do diálogo entre disciplinas e na reflexão acerca dessas problemáticas, além da análise de melhorias a serem implementadas. Um recurso possível é o desenvolvimento de capacitações voltadas aos profissionais da ESF, visando melhor identificação das questões de saúde mental e vulnerabilidade social (Leite et al., 2013). Tais questões estão em consonância com a proposta de Campos (2000), que refere que as práticas sociais devem ser organizadas democraticamente, estruturando-se de forma a permanecerem abertas a distintos campos de influência.

Nesse sentido, percebe-se que alguns estudos já identificam um trabalho em equipe interdisciplinar, por meio de efetivas discussões de casos entre as equipes e da definição de projetos terapêuticos compartilhados (Azevedo \& Kind, 2013; Leite et al., 2013). Essas ações 
promovem opiniões e saberes diversificados que se somam para o cuidado resolutivo do usuário, assim como na construção de práticas coletivas mais adequadas às necessidades da população. Contudo, identificou-se em algumas pesquisas que o usuário não era incluído enquanto agente ativo na construção do PTS (Azevedo \& Kind, 2013; Barros et al., 2015; Moura \& Luzio, 2014). Dessa forma, entende-se que essa prática não é exercida conforme seus pressupostos. Ainda, apontam-se dificuldades iniciais na implementação do PTS quanto ao estabelecimento das responsabilidades de cada profissional, o que indica uma necessidade de sistematização do processo de acompanhamento da equipe (Silva et al., 2016).

Outras ações previstas no âmbito do NASF são as práticas intersetoriais. A intersetorialidade é entendida como uma rede de organizações que estabelece acordos de cooperação, aliança e reciprocidade que propiciam reflexões e ações cotidianas em vários setores sociais conectados (Paula, Palha, \& Protti, 2004). Porém, cabe destacar que alguns estudos, apesar de indicarem a sua realização, constataram uma predominância de encaminhamentos e pedidos de pareceres realizados pelos serviços (Cela \& Oliveira, 2015; Ferro et al., 2014), o que perpetua uma dinâmica de trabalho baseada nos mecanismos de referência e contrarreferência. Nesse sentido, diferentes autores problematizam essa dinâmica, pois ela cria uma assistência fragmentada por meio da prática do encaminhamento, inviabilizando a criação de vínculo e de corresponsabilização entre os atores envolvidos (Cecilio, 1997; Zambenedetti \& Silva, 2008).

Outro importante fator a ser discutido é a formação de profissionais da saúde, dentre eles os psicólogos, já que muitos trabalhadores do NASF indicam déficits na sua graduação, principalmente em relação à perspectiva interdisciplinar e ao modelo de atenção à saúde do SUS (Azevedo \& Kind, 2013; Cela \& Oliveira, 2015; Leite et al., 2013). Esses achados vão ao encontro de estudos que analisam a atuação de psicólogos na atenção básica e apontam para as carências na formação acadêmica em relação ao trabalho a ser desenvolvido nas políticas públicas, o que faz com que, na maioria dos casos, ocorra transposição do modelo clínico para esse contexto (Andrade \& Simon, 2009; Böing \& Crepaldi, 2010; Dimenstein \& Macedo, 2012). 
Nesse sentido, estudos têm mostrado estratégias utilizadas em parcerias entre o Ministério da Saúde e universidades, a partir da criação de cursos de especialização e residências em saúde que visam a capacitação profissional para o trabalho da ESF. Iniciativas como o Programa de Educação pelo Trabalho para Saúde (PET-Saúde) e Programa Nacional de Reorientação da Formação em Saúde (Pró-Saúde) objetivam estimular mudanças no processo de formação, possibilitando uma atuação de caráter interdisciplinar em consonância com os princípios do SUS (Böing \& Crepaldi, 2010; Correia, Goulart, \& Furtado, 2017; Ferreira Neto, 2010). Outra estratégia potente é a educação permanente, a qual busca promover aprendizagem no e pelo trabalho, sendo balizada pela interdisciplinaridade e com ações direcionadas ao saber prático e transformador (Júnior \& Moreira, 2017).

Vale ressaltar que, apesar do esforço para identificar as práticas desenvolvidas pelos psicólogos no NASF, é necessário ter cautela para que não se produza fragmentação das funções exercidas pela equipe. Ao analisar as atividades que competem à psicologia ou outras áreas profissionais, produz-se um paradoxo ao buscar respostas na esfera intradisciplinar, quando a proposta do NASF é justamente a oposta (Correia et al., 2017). A iniciativa da política é estimular a colaboração entre saberes e profissões, extrapolando as competências e responsabilidades individuais para possibilitar articulação interdisciplinar.

\section{Considerações finais}

A partir desta revisão, percebe-se que a implementação do NASF ainda está em construção, mas vem avançando, no sentido de propor novas práticas e outra lógica de cuidado. É necessária uma mudança de cultura, tanto da população quanto dos profissionais, que pode ser sensibilizada por meio de capacitações e preparo das equipes que irão compor os NASF, assim como as ESF que irão recebê-los. Da mesma forma, é possível estimular trocas e reflexões através das discussões sobre o processo de trabalho, identificando fragilidades a serem superadas e potencialidades para uma nova forma de atuação. 
Em relação à prática da psicologia nesse contexto, foram encontrados resultados heterogêneos. Destaca-se o fato de a maioria dos artigos não descreverem claramente as atividades, o que dificultou a análise desses aspectos. Em parte das pesquisas, verificou-se uma atuação dos psicólogos incongruente com o contexto, por meio da transposição do modelo tradicional clínico para os serviços públicos de saúde. Sob outra perspectiva, artigos apontam para o desenvolvimento de ações interdisciplinares que consideram a complexidade da atenção básica e os princípios do SUS.

No que se refere às atividades desenvolvidas pelos psicólogos no NASF, o atendimento individual foi a principal ação atribuída especificamente a eles. Salienta-se que, apesar da importância dessa prática, essa não deve ser prioritária, tendo em vista a proposta do NASF. O valor da psicologia na perspectiva interdisciplinar está na escuta qualificada, que pode proporcionar a construção de intervenções e manejos condizentes com a complexidade das demandas da equipe de referência e da comunidade. As contribuições dessa área de saber devem transcender o olhar clínico individual, para contribuir nas diferentes atividades e na dinâmica de trabalho das equipes, colaborando na sensibilização das equipes e da comunidade. Esse pode ser um importante profissional para estimular as discussões acerca do trabalho das equipes e entre elas.

Vale salientar que, na esfera federal, a atual política de austeridade do SUS tende a dificultar esses processos que têm como objetivo a ampliação e o aprimoramento das ações desenvolvidas no sistema. Sanções, como a PEC 95, são barreiras político-institucionais que representam um retrocesso do investimento na saúde pública brasileira. Nesse sentido, ressalta-se a importância do cenário da Atenção Básica para a garantia de cuidado próximo às famílias e comunidades em situação de vulnerabilidade social, sendo estas as mais atingidas em momentos de crise política e econômica.

Entende-se que a política do NASF possui um caráter interdisciplinar. No entanto, como foi mostrado no presente estudo, é relevante analisar como cada disciplina - no caso, a psicologia - contribui na elaboração desse fazer. Uma potencialidade do estudo foi construir uma análise não apenas descritiva das ações, mas sim uma reflexão crítica de como vem sendo desenvolvidas. Postula-se que futuras pesquisas investiguem com maior 
aprofundamento a inserção da psicologia nas atividades interdisciplinares, tendo em vista a escassez de estudos que analisem suas práticas dentro do NASF.

Como limitações deste estudo, menciona-se a opção de trabalhar apenas com artigos, considerando que muitos estudos foram excluídos por não estarem publicados nesse formato. Ademais, outro fator limitador foi a utilização de descritor não indexado, visto que o NASF se configura como uma política do contexto brasileiro.

\section{Referências}

Andrade, J. F. S. D. M., \& Simon, C. P. (2009). Psicologia na atenção primária à saúde: reflexões e implicações práticas. Paidéia (Ribeirão Preto), 19(43), 167-175. https://doi.org/10.1590/S0103-863X2009000200005

Arce, V. A. R., \& Teixeira, C. F. (2017). Práticas de saúde e modelo de atenção no âmbito do Núcleo de Apoio à Saúde da Família em Salvador (BA). Saúde em Debate, 47(spe), 228 240. https://doi.org/10.1590/0103-11042017s317

Azevedo, N. S., \& Kind, L. (2013). Psicologia nos Núcleos de Apoio à Saúde da Família em Belo Horizonte. Psicologia: Ciência e Profissão, 33(3), 520-535. https://doi.org/10.1590/S1414-98932013000300002

Barros, J. de O., Gonçalves, R. M. de A., Kaltner, R. P., \& Lancman, S. (2015). Estratégia do apoio matricial: a experiência de duas equipes do Núcleo de Apoio à Saúde da Família (NASF) da cidade de São Paulo, Brasil. Ciência \& Saúde Coletiva, 20(9), 2847-2856. https://doi.org/10.1590/1413-81232015209.12232014

Böing, E., \& Crepaldi, M. A. (2010). O psicólogo na atenção básica: uma incursão pelas políticas públicas de saúde brasileiras. Psicologia: Ciência e Profissão, 30(3), 634-649. https://doi.org/10.1590/S1414-98932010000300014

Bonfim, I. G., Bastos, E. N. E., Góis, C. W. D. L., \& Tófoli, L. F. (2013). Apoio Matricial em Saúde Mental na Atenção Primária à Saúde: uma análise da produção científica e documental. Interface - Comunicação, Saúde, Educação, 17(45), 287-300. https://doi.org/10.1590/S1414-32832013005000012

Brasil, República Federativa do Brasil. Ministério da Saúde. (2008). Portaria $n^{\circ} 154$, de 24 de janeiro de 2008. Cria os Núcleos de Apoio à Saúde da Família (NASF). Brasília, DF: Ministério da Saúde. http://bvsms.saude.gov.br/bvs/saudelegis/gm/2008/prt0154_24_01_2008.html

Brasil, República Federativa do Brasil. Ministério da Saúde. (2011). Portaria $n^{\circ} 2.448$ de 21 de 
outubro de 2011 . Aprova a Política Nacional de Atenção Básica, estabelecendo a revisão de diretrizes e normas para a organização da Atenção Básica, para a Estratégia Saúde da Família (ESF) e o Programa de Agentes Comunitários de Saúde (PACS). Brasília, DF: Ministério da Saúde. http://bvsms.saude.gov.br/bvs/saudelegis/gm/2011/prt2488_21_10_2011.html

Brasil, República Federativa do Brasil. Ministério da Saúde. (2010). Cadernos de Atenção Básica, n. 27. Diretrizes do NASF: Núcleos de Apoio à Saúde da Família. Brasília, DF: Ministério da Saúde. http://189.28.128.100/dab/docs/publicacoes/cadernos_ab/abcad27.pdf

Brasil, República Federativa do Brasil. Ministério da Saúde. (2014). Cadernos de Atenção Básica, n. 39. Núcleo de Apoio à Saúde da Família - volume 1: Ferramentas para a gestão e para o trabalho cotidiano. Brasília, DF: Ministério da Saúde. http://189.28.128.100/dab/docs/portaldab/publicacoes/caderno_39.pdf

Brasil, República Federativa do Brasil. Ministério da Saúde. (2017). Portaria $n^{\circ}$ 2.436, de 21 de setembro de 2017. Aprova a Política Nacional de Atenção Básica, estabelecendo a revisão de diretrizes para a organização da Atenção Básica, no âmbito do Sistema Único de Saúde (SUS). Brasília, DF: Ministério da Saúde. http://bvsms.saude.gov.br/bvs/saudelegis/gm/2017/prt2436_22_09_2017.html

Campos, G. W. D. S. (2000). Saúde pública e saúde coletiva: campo e núcleo de saberes e práticas. Ciência \& Saúde Coletiva, 5(2), 219-230. https://doi.org/10.1590/S1413$\underline{81232000000200002}$

Campos, G. W. de S., \& Domitti, A. C. (2007). Apoio matricial e equipe de referência: uma metodologia para gestão do trabalho interdisciplinar em saúde. Cadernos de Saúde Pública, 23(2), 399-407. https://doi.org/10.1590/S0102-311X2007000200016

Campos, G. W. de S., Figueiredo, M. D., Pereira, N., \& Castro, C. P. (2014). A aplicação da metodologia Paideia no apoio institucional, no apoio matricial e na clínica ampliada. Interface: Communication, Health, Education, 18 (Supl 1), 983-995. https://doi.org/10.1590/1807-57622013.0324

Castro, C. P., \& Campos, G. W. S. (2014). Apoio institucional Paideia como estratégia para educação permanente em saúde. Trabalho, Educação e Saúde, 12(1), 29-50. https://doi.org/10.1590/S1981-77462014000100003

Cecilio, L. C. de O. (1997). Modelos tecno-assistenciais em saúde: da pirâmide ao círculo, uma possibilidade a ser explorada. Cadernos de Saúde Pública, 13(3), 469-478. https://doi.org/10.1590/S0102-311X1997000300022

Cela, M., \& Oliveira, I. F. de. (2015). O psicólogo no Núcleo de Apoio à saúde da Família: articulação de saberes e ações. Estudos de Psicologia, 20(1), 31-39. https://doi.org/10.5935/1678-4669.20150005

Correia, P. C. I., Goulart, P. M., \& Furtado, J. P. (2017). A avaliabilidade dos Núcleos de Apoio 
à Saúde da Família (Nasf). Saúde Em Debate, 47(spe), 345-359. https://doi.org/10.1590/0103-11042017s25

Cunha, G. T., \& Campos, G. W. de S. (2011). Apoio matricial e atenção primária em saúde. Saúde e Sociedade, 20(4), 961-970. https://doi.org/10.1590/S0104$\underline{12902011000400013}$

Dimenstein, M., \& Macedo, J. P. (2012). Formação em Psicologia. Psicologia: Ciência e Profissão, 32(esp), 232-245. https://doi.org/10.1590/S1414-98932012000500017

Dimenstein, M., Severo, A. K., Brito, M., Pimenta, A. L., Medeiros, V., \& Bezerra, E. (2009). O Apoio Matricial em Unidades de Saúde da Família: experimentando inovações em saúde. Saúde e Sociedade, 18(1), 63-74. https://doi.org/10.1590/S0104$\underline{12902009000100007}$

Ferreira Neto, J. L. (2010). A atuação do psicólogo no SUS: análise de alguns impasses. Psicologia: Ciência e Profissão, 30(2), 390-403. https://doi.org/10.1590/S1414$\underline{98932010000200013}$

Ferro, L. F., Silva, E. C., Zimmermann, A. B., Castanharo, R. C. T., \& Oliveira, F. R. L. (2014). Interdisciplinaridade e intersetorialidade na estratégia saúde da família e no núcleo de apoio à saúde da família: Potencialidades e desafios. Mundo Da Saude, 38(2), 129-138. https://doi.org/10.15343/0104-7809.20143802129138

Gonçalves, R. M. de A., Lancman, S., Sznelwar, L. I., Cordone, N. G., Barros, J. de O., Gonçalves, R. M. de A. et al. (2015). Estudo do trabalho em Núcleos de Apoio à Saúde da Família (NASF), São Paulo, Brasil. Revista Brasileira de Saúde Ocupacional, 40(131), 5974. https://doi.org/10.1590/0303-7657000078013

Hori, A. A., \& Nascimento, A. de F. (2014). O Projeto Terapêutico Singular e as práticas de saúde mental nos Núcleos de Apoio à Saúde da Família (NASF) em Guarulhos (SP), Brasil. Ciência \& Saúde Coletiva, 19(8), 3561-3571. https://doi.org/10.1590/141381232014198.11412013

Júnior, J. P. B. \& Moreira, D. C. (2017). Educação permanente e apoio matricial: formação, vivências e práticas dos profissionais dos Núcleos de Apoio à Saúde da Família e das equipes apoiadas. Cadernos de Saúde Pública, 33(9), 1-13. https://doi.org/10.1590/0102-311×00108116

Klein, A. P., \& d'Oliveira, A. F. P. L. (2017). O "cabo de força" da assistência: concepção e prática de psicólogos sobre o Apoio Matricial no Núcleo de Apoio à Saúde da Família. Cadernos de Saúde Pública, 33(1), 1-10. https://doi.org/10.1590/0102-311×00158815 Lancman, S., Gonçalves, R. M. de A., Cordone, N. G., \& Barros, J. de O. (2013). Estudo do trabalho e do trabalhar no Núcleo de Apoio à Saúde da Família. Revista de Saúde Pública, 47(5), 968-975. https://doi.org/10.1590/S0034-8910.2013047004770

Leite, D. C., Andrade, A. B., \& Bosi, M. L. M. (2013). A inserção da Psicologia nos Núcleos de 
Apoio à Saúde da Família. Physis: Revista de Saúde Coletiva, 23(4), 1167-1187. https://doi.org/10.1590/S0103-73312013000400008

Macedo, M. P. V., Guimarães, J. M. X., Sampaio, J. J. C., Morais, A. P. P., \& Carneiro, C. (2016). Análise do processo de trabalho no núcleo de apoio à saúde da família em um município do nordeste brasileiro. Revista Gerencia y Políticas de Salud, 15(30), 194-211. http://dx.doi.org/10.11144/Javeriana.rgyps15-30.aptn

Moura, R. H., \& Luzio, C. A. (2014). O apoio institucional como uma das faces da função apoio no Núcleo de Apoio à Saúde da Família (NASF): Para além das diretrizes. Interface: Communication, Health, Education, 18(Supl 1), 957-970. https://doi.org/10.1590/1807-57622013.0333

Nascimento, D. do, \& Oliveira, M. A. D. C. (2010). Reflexões sobre as competências profissionais para o processo de trabalho nos Núcleos de Apoio à Saúde da Família. $O$ Mundo da Saúde, 34(1), 92-96. http://www.saocamilosp.br/pdf/mundo_saude/74/12_revisao_reflexoes.pdf

Oliveira, I. F., Amorim; K. M. O., Paiva, R. A., Oliveira, K. S. A., Nascimento, M. N. C., \& Araújo, R. L. (2017). A atuação do psicólogo nos NASF: desafios e perspectivas na atenção básica. Temas em Psicologia, 25(1), 291-304. https://dx.doi.org/10.9788/TP2017.1-17Pt

Paula, K. A. de P., Palha, P. F., \& Protti, S. T. P. (2004). Intersetorialidade: uma vivência prática ou um desafio a ser conquistado? Interface, 8(15), 331-348. http://doi.org/10.1590/S1414-32832004000200011

Perrella, A. C. (2015). A experiência da Psicologia no NASF: capturas, embates e invenções. Gerais: Revista Interinstitucional de Psicologia, 8(2), 443-452. http://pepsic.bvsalud.org/pdf/gerais/v8n2/v8n2a12.pdf

Pinto, D. M., Jorge, M. S. B., Pinto, A. G. A., Vasconcelos, M. G. F., Cavalcante, C. M., Flores, A. Z. T. et al. (201 1). Projeto terapêutico singular na produção do cuidado integral: Uma construção coletiva. Texto e Contexto Enfermagem, 20(3), 293-302. https://doi.org/10.1590/S0104-07072011000300010

Ribeiro, M. D. A., Bezerra, E. M. A., Costa, M. S., Branco, C. E. C., Neto, J. D. A., Moreira, A. K. F. et al. (2014). Avaliação da atuação do núcleo de apoio à saúde da família. Revista Brasileira em Promoção da Saúde, 27(2), 224-231. https://doi.org/10.5020/18061230.2014.p224

Santana, J. S., Azevedo, T. L., Reichert, A. D. S., Medeiros, A. L., \& Soares, M. J. G. O. (2015). Center for family health support: team performance at the family health strategy. Revista de Pesquisa: Cuidado É Fundamental Online, $7(2), \quad 2362-2371$. https://doi.org/10.9789/2175-5361.2015.v7i2.2362-2371

Santeiro, T. V. (2012). Processos Clínicos em Núcleos de Apoio à Saúde da Família/NASF: Estágio Supervisionado. Psicologia: Ciência e Profissão, 32(4), 942-955. 
https://doi.org/10.1590/S1414-98932012000400013

Santos, R. A. B. G., Figueiredo, R. L. C., \& Lima, L. C. (2017). Apoio matricial e ações na atenção primária: experiência de profissionais de Esf e Nasf. Saúde em Debate, 47(114), 694-706. https://doi.org/10.1590/0103-1104201711402

Silva, A. I., Loccioni, M. F. L., Orlandini, R. F., Rodrigues, J., Peres, G. M., \& Maftum, M. A. (2016). Projeto Terapêutico Singular Para Profissionais Da Estratégia De Saúde Da Família. Revista Cogitare Enfermagem, 21(3), 1-8. http://doi.org/10.5380/ce.v21i3.45437

Silva, A. T. C. da, Aguiar, M. E., Winck, K., Rodrigues, K. G. W., Sato, M. E., Grisi, S. J. F. E. et al. (2012). Núcleos de Apoio à Saúde da Família: desafios e potencialidades na visão dos profissionais da Atenção Primária do Município de São Paulo, Brasil. Cadernos de Saúde Pública, 28(11), 2076-2084. https://doi.org/10.1590/S0102-311X2012001100007

Soares, S., \& De Oliveira, W. F. (2016). The matrix approach to mental health care: Experiences in Florianopolis, Brazil. Journal of Health Psychology, 21(3), 336-345. https://doi.org/10.1177/1359105316628752

Sousa, D. de, Oliveira, I. F. de, \& Costa, A. L. F. (2015). Entre o especialismo e o apoio: psicólogos no Núcleo de Apoio à Saúde da Família. Psicologia USP, 26(3), 474-483. https://doi.org/10.1590/0103-656420140059

Sundfeld, A. C. (2010). Clínica ampliada na atenção básica e processos de subjetivação: Relato de uma experiência. Physis, 20(4), 1079-1097. https://doi.org/10.1590/S010373312010000400002

Vasconcelos, F. G., \& Aléssio, R. L. dos S. (2019). Construções Identitárias de Psicólogos em NASF: Reflexões para a Prática Profissional. Psicologia: Ciência e Profissão, 39, e174637, 1-15. https://doi.org/10.1590/1982-3703003174637

Zambenedetti, G., \& Silva, R. (2008). A noção de rede nas reformas sanitária e psiquiátrica no $\begin{array}{llll}\text { Brasil. Psicologia } & \text { 14(1), Revista, 131-150. }\end{array}$ http://pepsic.bvsalud.org/scielo.php?pid=S1677$11682008000100008 \&$ script $=$ sci_arttext 